\title{
VEGF reverts the cognitive impairment induced by a focal traumatic brain injury during the development of rats raised under environmental enrichment
}

\author{
N. Ortuzar ${ }^{\mathrm{a}, *}$, I. Rico-Barrio $^{\mathrm{a}}$, H. Bengoetxea ${ }^{\mathrm{a}}$, E.G. Argandoña ${ }^{\mathrm{b}}$, J.V. Lafuente ${ }^{\mathrm{a}}$ \\ a Laboratory of Clinical and Experimental Neuroscience (LaNCE), Department of Neuroscience, Faculty of Medicine and Odontology, University of the \\ Basque Country (UPV/EHU), Barrio Sarriena, E-48940 Leioa, Spain \\ ${ }^{\mathrm{b}}$ Department of Medicine, Unit of Anatomy, University of Fribourg. Rue Albert Gockel 1, CH-1700 Fribourg Switzerland
}

\section{Introduction}

Growth factors and its receptors are key regulators for central nervous system development as well as for homeostasis maintenance. These molecules exert effects over all components of the neurogliovascular unit (NVU), such as vessels, neurons and glia,

* Corresponding author at: Laboratory of Clinical and Experimental Neuroscience (LaNCE). Department of Neuroscience. Faculty of Medicine and Odontology. University of the Basque Country (UPV/EHU). Barrio Sarriena s/n. E48940, Leioa, Spain. Tel.: +3494601 5606; fax: +34946015055.

E-mail address: naiara.ortuzar@ehu.es (N. Ortuzar). and some authors refer to these molecules as angioglioneurins $[1,2]$. Vascular Endothelial Growth Factor (VEGF) is the archetypal angioglioneurin and acts on both vascular and neural development.

The role of VEGF in the nervous system is extensive. Apart from its angiogenic effect in developmental and pathological angiogenesis [3], it has been described as a neuroprotective, neurotrophic and neurogenic molecule [4-7]. The neuroprotective function of VEGF appears to be due to a combination of direct neuroprotective effects and the stimulation of angiogenesis. In addition, previous studies have described its neurogenic effect in the subventricular zone (SVZ) [8] and in the subgranular zone (SGZ) of the dentate gyrus [9]. It has been postulated that VEGF promotes the proliferation and differentiation of neuronal precursors, releasing neurotrophic factors 
[10] or exerting a direct mitogenic action on neural precursors [11]. Further studies described VEGF as a direct mediator of improved cognition in rodents [12-14] and as inducing long-term synaptic enhancement in hippocampal neurons [15]. Nonetheless, a recent study has shown that VEGF promotes hippocampus-dependent memory independently of its effects on neurogenesis and angiogenesis, by increasing the synaptic strength [16].

Enriched environment (EE) is an experimental paradigm defined as "a combination of complex and inanimate social stimulation" [17] that induces cellular, molecular and behavioural effects, not only in standard conditions but also in pathological ones $[18,19]$. Whereas initial studies showed that EE modified cortical weight [20,21], subsequent studies described an increase of nuclear and soma size in neurons, dendritic branching or synapsis size [22-24] among other effects. Moreover, EE also increases neurogenesis in the hippocampus [13] as it increases the expression of angioglioneurins such as VEGF or BDNF [25-27]. Therefore, EE has strong effects on the plasticity of neural connections, especially in the visual cortex [28].

The postnatal development of the visual cortex is modulated by environmental experience. Sensory modifications during an early critical period result in substantial plasticity and are a crucial factor in establishing the mature circuitry [29]. In rats, the critical period for the visual system is located between the third and the fifth postnatal weeks and the maximum peak of experience-induced changes occurs during the fourth and the fifth weeks [30,31].

The aim of the present study was to investigate the neurovascular and cognitive effects of VEGF infusion and enriched environment during the critical period of the visual cortex in developing rats.

\section{Material and methods}

\subsection{Animals and housing}

10 Long-Evans rats (Harlan, Barcelona, Spain) were used for each experimental group. Animals were raised in different rearing conditions:

1. Standard conditions (SC): rats raised in a standard laboratory cage $(500 \mathrm{~mm} \times 280 \mathrm{~mm} \times 140 \mathrm{~mm})$ with 12 -h light/dark cycle (lights on at 8 a.m.)

2. Enriched environment (EE): rats raised in a large cage $(720 \mathrm{~mm} \times 550 \mathrm{~mm} \times$ $330 \mathrm{~mm}$ ) furnished with colourful toys and differently shaped objects (shelters, tunnels) that were changed every 2 days (with 12-h light/dark cycle; lights on at 8 a.m.).

\subsection{Minipump implantation}

Experiments were performed on Long-Evans rats $72 \mathrm{~h}$ prior to the beginning of the critical period (P18, 30-40 g). Animals were anaesthetized with avertin $(1 \mathrm{ml} / 100 \mathrm{~g})$ and a sagittal incision was made midway between the eyes. Firstly, the skin was retracted, and then the periosteum. Subsequently a subcutaneous pocket was opened in the animal's back into which the osmotic minipump (Mod. 1004, Alzet, Cupertino, CA, USA) was inserted. A brain infusion kit (Mod. Alzet Brain Infusion Kit III, Alzet) was fixed to the skull with cyanoacrylate. Minipumps were connected via PVC tubing to the infusion kit and the cannula was implanted $1 \mathrm{~mm}$ lateral to the sagittal suture and $1 \mathrm{~mm}$ anterior to lambda into the left hemisphere. Total operating time was approximately $25 \mathrm{~min}$.

Different experimental groups were used:

a) A group of non-operated rats as control

b) PBS infusion

c) VEGF infusion

VEGF (Ref: sc-4571, Santa Cruz Biotechnology Inc, Germany) was administered $(100 \mathrm{ng} / \mathrm{ml})$ at a delivery rate of $0.11 \mu \mathrm{l} / \mathrm{h}$ with the cannula placed in the middle cortical layers. Within each group, the following visual stimulation conditions were studied:

SC-SC: Rats raised in standard conditions (SC) before and after minipump implantation.

SC-EE: Rats raised in standard conditions (SC) until minipump implantation (P18) and in an enriched environment (EE) after implantation until P46.
After surgery had been completed, food and water were provided ad libitum. Minipumps were left in position for four weeks until P46 (150-200 g).

\subsection{Ethics statements}

All animal experiments were performed in accordance with the European Community Council Directive (2010/63/EU) and approved by the Ethics Committee for Animal Welfare (CEBA) of the University of the Basque Country.

\subsection{Morris water maze}

Spatial learning and memory were assessed in a Morris water maze task during the last 11 days of the infusion period. In this task, animals learned to locate the position of the submerged platform using extra-maze spatial cues.

The water maze consisted of a circular swimming pool, $170 \mathrm{~cm}$ diameter and $0.6 \mathrm{~m}$ height, filled with water $\left(22 \pm 1^{\circ} \mathrm{C}\right)$ made opaque with non-toxic white paint. Visual cues were fixed on the walls constantly visible from the pool, which was conceptually divided into four quadrants and had four points designed as starting points (north, south, east and west). During five days (days 1-5), animals were tested for place-learning acquisition with the escape platform ( $11 \mathrm{~cm}$ diameter, $47 \mathrm{~cm}$ height) located in the middle of the southeast quadrant, $2 \mathrm{~cm}$ below water surface. Four trials per day were performed ( $30 \mathrm{~min}$. inter-trial interval), introducing the rats randomly from each of the four starting positions while facing the wall, and allowing them to swim until they located the platform. Animals that failed to find the platform within $120 \mathrm{~s}$ were guided to the platform and left there for $60 \mathrm{~s}$, as were the successful ones. On the 7th day, the platform was removed and a single probe trial was performed $(120 \mathrm{~s})$ to assess spatial memory retention for the platform location. Time spent in the target area as well as in the other quadrants was measured. Next day, a session with a cued visible platform was carried out in which the platform was marked by a black flag as a control procedure to discard motor or motivational sensory deficits between experimental groups. Finally, during days 9-11, animals were subjected to a reversal test. The location of the hidden platform was changed to northeast and animals were tested for place-learning acquisition of the new location. All the trials were recorded and traced with an image tracking system (SMART, Panlab SL, Barcelona, Spain) connected to a video camera placed above the pool. The latency and travelled path length to find the platform were measured in each trial and the mean value for each day was calculated.

\subsection{Fixation and tissue processing}

Rats were anaesthetized with $6 \%$ chloral hydrate. After anaesthesia, the cannula was removed and the animals were transcardially perfused with a saline solution followed by a fixative containing $4 \%$ paraformaldehyde in $0.1 \mathrm{M}$ phosphatebuffered saline solution (PBS). After perfusion, brains were stored overnight at $4{ }^{\circ} \mathrm{C}$ in fresh fixative. The next day, the brain was stored in 30\% sucrose in $0.1 \mathrm{M}$ PBS until the tissues sank. Then, samples were coronally cut at $50 \mu \mathrm{m}$ with a cryotome and serially-collected sections were kept in $0.1 \mathrm{M}$ PBS to be histochemically and immunohistochemically processed using the free-floating method.

\subsection{Butyryl cholinesterase histochemistry}

Sections were histochemically processed for Butyryl Cholinesterase to visualize the vascular pattern. Sections were washed twice in $0.1 \mathrm{M}$ Tris-maleate buffer (TMB) (pH 6), acetylcholinesterase was inhibited in a BW284CS1 (1,5-bis(4-allyldimethylammoniumphenyl)-pentan-3-one dibromide) (Ref: A-9013, Sigma-Aldrich, Spain) $0.05 \mathrm{M} 20 \mathrm{~min}$ bath and sections were incubated overnight in the following incubation solution: butyryl thiocholine iodide (Ref: 108150250, Acros Organics, Barcelona, Spain) $1 \mathrm{mg} / \mathrm{ml}, 5 \%$ sodium citrate $0.1 \mathrm{M}, 10 \%$ copper sulphate $30 \mathrm{mM}$, $10 \%$ BW284CS1 $0.05 \mathrm{mM}, 10 \%$ potassium ferricyanide $5 \mathrm{mM}$ and $65 \% \mathrm{TMB} 0.1 \mathrm{M}$. The next day, sections were washed in TMB, mounted on gelatine-coated slides, dehydrated and covered.

\subsection{Immunohistochemistry}

Sections were immunohistochemically processed for NeuN (Ref: MAB377, Chemicon International Inc, Spain, 1:400). Firstly, sections were washed in 0.1 M PBS and then incubated with $4 \% \mathrm{H}_{2} \mathrm{O}_{2}$ /methanol for 20 min in order to quench endogenous peroxidase activity. Afterwards, non-specific sites were blocked by adding BSA $5 \%$ in the incubation medium for $1 \mathrm{~h}$. Sections were then incubated overnight at $4{ }^{\circ} \mathrm{C}$ with primary antibody. The following day, sections were washed in $0.1 \mathrm{M}$ PBS and incubated with biotinylated secondary antibodies (Elite ABC kit, Vector Laboratories, Burlingame, CA, USA). The immunohistochemical reaction was revealed by the avidin-biotin complex (Elite ABC kit, Vector Laboratories) using diaminobenzidine as a chromogen. Sections were mounted on gelatine-coated slides, dehydrated and covered.

\subsection{LEA lectin staining and double-label immunofluorescence}

Free-floating sections of primary visual cortex and the dentate gyrus were used for both LEA lectin staining and double-label immunofluorescence. For microvessel staining, Lycopersicon esculentum (tomato lectin LEA) histochemistry 


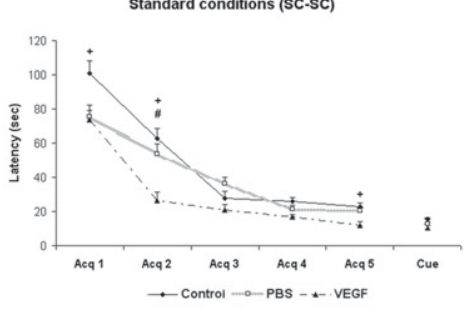

C

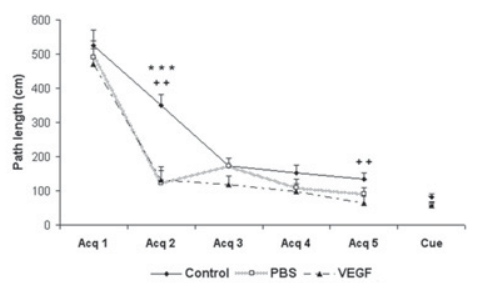

e

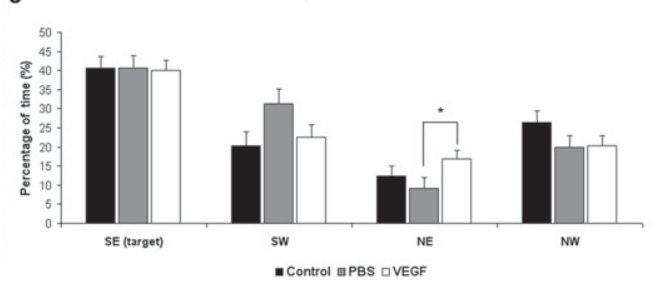

g

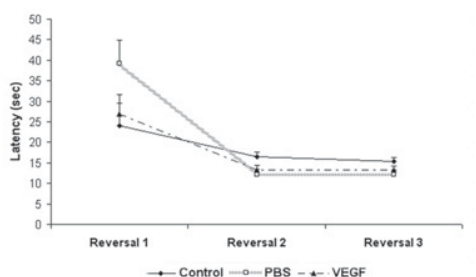

b

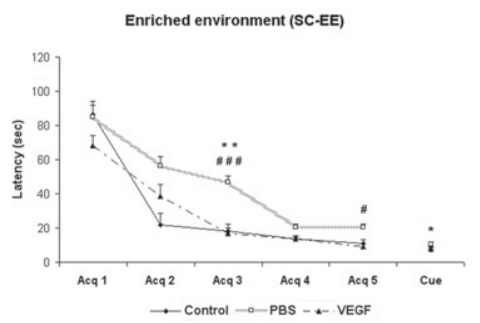

d
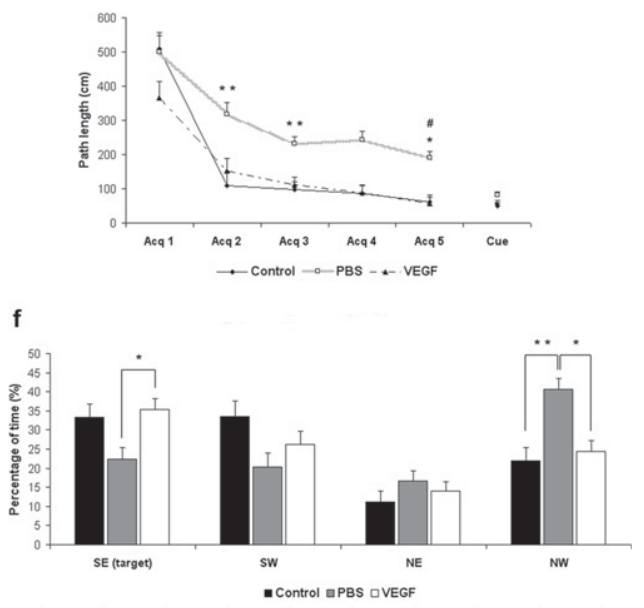

h

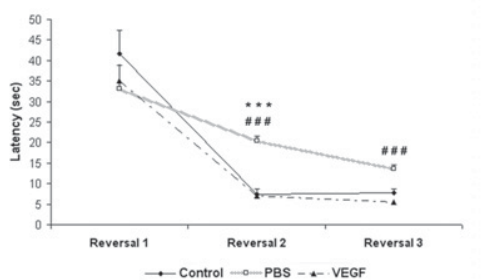

Fig. 1. Effects of VEGF administration on spatial learning and memory in the Morris Water Maze task of animals reared in standard conditions (SC-SC) and in enriched environment (SC-EE). 5 acquisitions, 1 cued, 1 removal and 3 reversal sessions were performed in different experimental groups (Control, PBS and VEGF). Escape latencies (a, b) and travelled path length (c, d) to reach the platform during the learning process. Percentage of time spent in each quadrant when platform was removed (e, f). Escape latency $(\mathrm{g}, \mathrm{h})$ to reach the platform during the reversal test. Mean \pm SEM, $n=10 .{ }^{+}$Significance between VEGF and Control $\left({ }^{+} p<0.05 ;{ }^{++} p<0.01\right)$. ${ }^{*}$ Significance between Control and PBS $\left({ }^{*} p<0.05 ;{ }^{* *} p<0,01 ;{ }^{* * *} p<0.001\right)$. ${ }^{*}$ Significance between VEGF and PBS $\left({ }^{\#} p<0.05 ;{ }^{* \# \#} p<0.001\right)$.

was used. Sections were washed in 0.1 M PBS ( $\mathrm{pH}$ 7.4). Then, non-specific sites were blocked by adding BSA 4\% in the incubation medium for $1 \mathrm{~h}$ followed by overnight incubation with LEA-TRICT antibody (Ref: L9511, Sigma-Aldrich, Spain, 1:200) in $1 \% \mathrm{BSA}$ in $0.1 \mathrm{M}$ PBS containing $0.5 \%$ Triton X-100. Afterwards, sections were washed in PBS. Double immunolabelling using GFAP (glial fibrillary acidic protein), NeuN and LEA as markers for astrocytes, neurons and endothelial cells, respectively, and for VEGF were carried out to determine co-localization with each cell type. Sections were washed in 0.1 M PBS and were incubated with blocking solution (5\% BSA in $0.1 \mathrm{M}$ PBS) for $1 \mathrm{~h}$ followed by overnight incubation with a cocktail of primary antibodies in $1 \%$ BSA in $0.1 \mathrm{M}$ PBS containing $0.5 \%$ Triton X-100. The antibodies used were monoclonal mouse anti-GFAP (Ref: G-3893, Sigma-Aldrich, Spain, 1:400), monoclonal mouse anti-NeuN (Ref: MAB377, Chemicon International Inc, 1:400), and polyclonal rabbit anti-VEGF (Ref: sc-152, Santa Cruz Biotechnology, Inc., USA, 1:400). After rinsing, sections were incubated for $2 \mathrm{~h}$ with the following fluorochrome conjugated secondary antibodies (goat antimouse Alexa Fluor ${ }^{\circledR} 488$, goat antirabbit Alexa Fluor ${ }^{\circledR}$ 568; Invitrogen, Spain, 1:400) in 1\% BSA in 0.1 M PBS containing $0.3 \%$ Triton X-100. Hoechst-33258 was added to counterstain the nuclei. Sections were rinsed, mounted on gelatine-coated slides, and cover-slipped in aqueous medium. For all methods, negative controls in which the primary antibodies were omitted were included in each staining run. Images were acquired for confocal fluorescence microscopy with an Olympus Fluoview FV500 confocal microscope using sequential acquisition to avoid overlapping of fluorescence emission spectra.

\subsection{Morphometric procedures}

Vascular and neuronal densities were measured by the optical dissector method in Butyryl Cholinesterase histochemistry and NeuN immunohistochemistry, respectively, with the aid of the Mercator Image Analysis system (Explora Nova, La
Rochelle, France). For this purpose, probes of $50 \mu \mathrm{m} \times 50 \mu \mathrm{m}$ separated by $120 \mu \mathrm{m}$ were launched into a previously-delimited area corresponding to layer IV of the primary visual cortex and the granular cell layer of the dentate gyrus. Positive cells and vessels were counted if found to be present inside the probe and if they did not touch the forbidden $X$ and $Y$ axes. 10 animals and 10 histological sections per animal were used for each experimental group, and 2 measurements were taken for each section (ipsilateral and contralateral hemisphere), making a total of 200 measurements for each group. Measurements of each slice of the cortex were taken and the mean value per animal was calculated.

\subsection{Statistical analysis}

Statistical analysis was performed using SPSS statistical software (version 19.0, IBM, Spain). Prior to analysis, data were examined for normal distribution using the Kolmogorov-Smirnov test and for homogeneity of variances using Levene's test. The effects of experimental conditions were evaluated using the one-way ANOVA analysis with the Bonferroni correction for equal variances or Tamhane's T2 correction for unequal variances. Data are described as mean \pm SEM. Significance was declared at $p<0.05$.

\section{Results}

\subsection{Effects of VEGF administration in learning and memory}

\subsubsection{Standard conditions (SC-SC)}

Spatial memory acquisition testing showed that the escape latency as well as total path length of the VEGF-infused group were 
Table 1

Effect of enriched environment on spatial learning and memory in each experimental group (Control, PBS, VEGF). Mean value of escape latency and travelled path length \pm SEM and $p$ value between environmentally-enriched (SC-EE) and standard condition (SC-SC) reared groups within each experimental group.

\begin{tabular}{|c|c|c|c|c|c|c|}
\hline & \multicolumn{2}{|l|}{ Control } & \multicolumn{2}{|l|}{ PBS } & \multicolumn{2}{|l|}{ VEGF } \\
\hline & \multicolumn{2}{|l|}{ SC-EE vs. SC-SC } & \multicolumn{2}{|l|}{ SC-EE vs. SC-SC } & \multicolumn{2}{|l|}{ SC-EE vs. SC-SC } \\
\hline & Latency (s) & $p$ & Latency (s) & $p$ & Latency (s) & $p$ \\
\hline Acq 1 & $86 \pm 7$ vs. $101 \pm 7$ & 0.863 & $84 \pm 7$ vs. $75 \pm 7$ & 0.988 & $68 \pm 5$ vs. $73 \pm 5$ & 1.000 \\
\hline Acq 2 & $22 \pm 6$ vs. $62 \pm 6$ & 0.000 & $56 \pm 5$ vs. $53 \pm 6$ & 1.000 & $38 \pm 7$ vs. $26 \pm 5$ & 0.886 \\
\hline Acq 3 & $18 \pm 4$ vs. $27 \pm 4$ & 0.227 & $46 \pm 3$ vs. $36 \pm 3$ & 0.940 & $17 \pm 3$ vs. $20 \pm 3$ & 0.951 \\
\hline Acq 4 & $13 \pm 2$ vs. $26 \pm 2$ & 0.003 & $20 \pm 2$ vs. $21 \pm 2$ & 1.000 & $13 \pm 1$ vs. $16 \pm 1$ & 0.795 \\
\hline Acq 5 & $11 \pm 2$ vs. $22 \pm 2$ & 0.001 & $20 \pm 2$ vs. $20 \pm 2$ & 1.000 & $9 \pm 1$ vs. $12 \pm 2$ & 0.795 \\
\hline Cue & $7 \pm 1$ vs. $15 \pm 1$ & 0.002 & $10 \pm 1$ vs. $12 \pm 1$ & 0.999 & $8 \pm 1$ vs. $10 \pm 1$ & 0.510 \\
\hline $\operatorname{Rev} 1$ & $41 \pm 5$ vs. $24 \pm 5$ & 0.427 & $33 \pm 5$ vs. $39 \pm 5$ & 0.994 & $35 \pm 4$ vs. $27 \pm 4$ & 0.096 \\
\hline Rev 2 & $7 \pm 1$ vs. $16 \pm 1$ & 0.000 & $20 \pm 1$ vs. $12 \pm 1$ & 0.035 & $7 \pm 1$ vs. $13 \pm 1$ & 0.033 \\
\hline \multirow[t]{4}{*}{$\operatorname{Rev} 3$} & $7 \pm 1$ vs. $15 \pm 1$ & 0.000 & $13 \pm 1$ vs. $12 \pm 1$ & 0.784 & $5 \pm 0.8$ vs. $13 \pm 1$ & 0.000 \\
\hline & \multicolumn{2}{|l|}{ Control } & \multicolumn{2}{|l|}{ PBS } & \multicolumn{2}{|l|}{ VEGF } \\
\hline & \multicolumn{2}{|l|}{ SC-EE vs. SC-SC } & \multicolumn{2}{|l|}{ SC-EE vs. SC-SC } & \multicolumn{2}{|l|}{ SC-EE vs. SC-SC } \\
\hline & Latency (s) & $p$ & Latency (s) & $p$ & Latency (s) & $p$ \\
\hline Acq 1 & $508 \pm 48$ vs. $525 \pm 46$ & 1.000 & $501 \pm 46$ vs. $490 \pm 48$ & 1.000 & $367 \pm 46$ vs. $471 \pm 46$ & 0.169 \\
\hline Acq 2 & $110 \pm 37$ vs. $350 \pm 30$ & 0.000 & $319 \pm 33$ vs. $122 \pm 38$ & 0.008 & $153 \pm 36$ vs. $131 \pm 38$ & 1.000 \\
\hline Acq 3 & $98 \pm 23$ vs. $139 \pm 22$ & 0.494 & $232 \pm 21$ vs. $173 \pm 22$ & 0.931 & $112 \pm 21$ vs. $118 \pm 25$ & 1.000 \\
\hline Acq 4 & $86 \pm 25$ vs. $153 \pm 23$ & 0.013 & $244 \pm 24$ vs. $110 \pm 24$ & 0.246 & $89 \pm 22$ vs. $97 \pm 24$ & 1.000 \\
\hline Acq 5 & $61 \pm 21$ vs. $133 \pm 19$ & 0.001 & $192 \pm 18$ vs. $90 \pm 19$ & 0.225 & $57 \pm 19$ vs. $64 \pm 20$ & 0.998 \\
\hline Cue & $49 \pm 9$ vs. $82 \pm 9$ & 1.000 & $82 \pm 8$ vs. $58 \pm 10$ & 1.000 & $57 \pm 8$ vs. $57 \pm 9$ & 0.812 \\
\hline
\end{tabular}

decreased compared to non-operated control groups and the group infused with PBS during the learning process (Fig. 1a, c). The VEGFinfused group showed statistically-significant differences in escape latency compared to non-operated control group (Acq 1, $p=0.005$ Acq 2, $p=0.001$; Acq 5, $p=0.003$ ) and the PBS-infused group (Acq $2, p=0.009$ ) during the acquisition phase. The VEGF-infused group also showed a diminished total path length compared to the nonoperated control group (Acq 2, $p=0.001$; Acq 5, $p=0.002$ ), as did the non-operated control group compared to the PBS-infused group (Acq 2, $p=0.001$ ). The probe trial was performed $24 \mathrm{~h}$ after the final day of training and no differences between experimental groups were observed in the target quadrant $(40 \%$ in the control, PBSinfused and VEGF-infused groups; $p=1.000$ ) (Fig. 1e). In addition, when the platform location was changed, no statistical differences were found in escape latencies between the studied groups (Fig. 1g).

\subsubsection{Enriched environment (SC-EE)}

The VEGF-infused group showed statistically-decreased escape latency and total path length compared to the PBS-infused group during the learning process (Fig. 1b, d). Between the third and fifth days of acquisition, the VEGF-infused group presented lower latency compared to the PBS-infused group $(p=0.000$ and $p=0.010$ respectively). Differences in path length were also statistically significant (Acq 3, $p=0.037$ and Acq 5, $p=0.022$ ). In addition, the non-operated control group also showed statistically-significant lower latency than the PBS-infused group in the third day of acquisition $(p=0.001)$, whereas the differences in path length were significant during the second $(p=0.002)$, third $(p=0.009)$ and fifth day $(p=0.029)$ of the learning process. On the cued visible platform day, the PBS-infused group also showed a statisticallysignificant difference compared to the control group in escape latency $(p=0.026)$ but no differences were observed in total path length. Furthermore, in the probe trial performance, the PBSinfused group spent less time in the target quadrant than the VEGF-infused group and the non-operated control group (33\% control, 22\% PBS-infused and 35\% VEGF-infused group; $p=0.043$ and $p=0.295$ respectively) (Fig. 1f). When the platform location was changed, the VEGF-infused and control groups also presented significantly lower escape latency than the PBS-infused group $(p=0.000$ in both cases) (Fig. $1 \mathrm{~h})$.

\subsubsection{Enriched environment (SC-EE) vs. standard conditions (SC-SC)}

During the learning process, animals reared in enriched environment showed statistically-decreased latency compared to standard-reared animals in the non-operated control group (Acq 2, $p=0.000$; Acq 4, $p=0.003$; Acq 5, $p=0.001$ and Cue, $p=0.002$ ). Path length was also statistically diminished in enriched animals compared to the control group that was standard-reared during the learning process (Acq 2, $p=0.000$; Acq 4, $p=0.013$; Acq 5, $p=0.001$ ). In the PBS and VEGF-infused groups, no differences in the escape latency between SC-EE and SC-SC reared rats were found. In contrast, in the PBS-infused group, the path length for enriched rats was significantly longer than in standards during the second day of the acquisition phase $(p=0.008)$. In addition, whereas in the PBS-infused group the probe trial performance showed statistically-significant lower time spent in the target quadrant of SC-EE reared rats $(40 \%$ in SC-SC vs. $22 \%$ in SC-EE; $p=0.002)$, no significant differences were found in nonoperated controls (40\% in SC-SC and 33\% in SC-EE, $p=1.000$ ) or the VEGF-infused groups (40\% in SC-SC and 35\% in SC-EE; $p=1.000$ ). Furthermore, in the non-operated control group, SC-EE reared rats also showed lower latency during the reversal test compared to SC-SC reared rats $(p=0.000)$. The same results were obtained in the VEGF-infused group $(p=0.033$ and $p=0.000)$. In contrast, in the PBS-infused group, lower escape latency was observed in the SC-SC reared group during the reversal learning process $(p=0.035)$ (Table 1).

\subsection{Effects of VEGF administration in the dentate gyrus}

\subsubsection{Standard conditions (SC-SC)}

The vascular density of the dentate gyrus showed no differences among the studied experimental groups. In the ipsilateral cortex, the PBS-infused group showed 3\% lower density compared to the non-operated group, while in the VEGF-infused group, observed density was $3 \%$ higher ( $p=1.000$ in both cases). The difference between the PBS-and VEGF-infused groups was $7 \%(p=1.000)$, being higher in the latter one. Similar results were found in the contralateral hemisphere. Whereas the PBS-infused group presented $5 \%(p=1.000)$ lower vascular density, the VEGF-infused group 

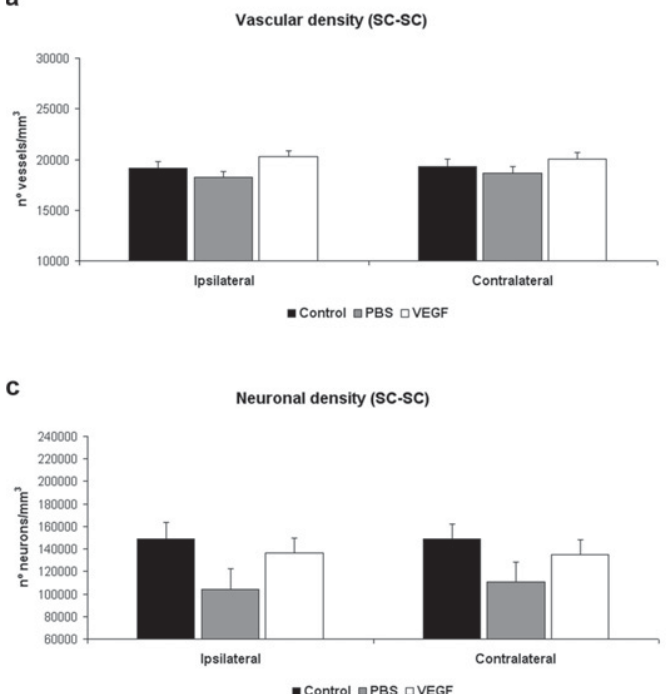

b

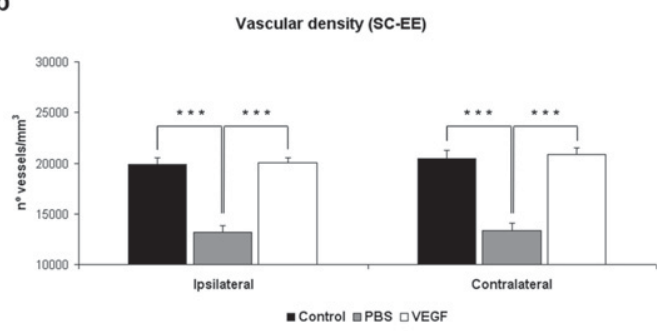

d

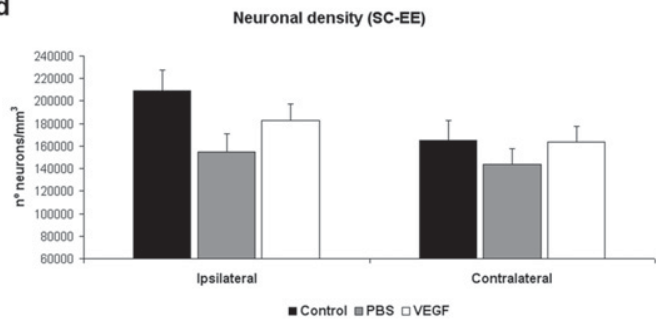

Fig. 2. Effects of VEGF administration on vascular and neuronal densities in the dentate gyrus. Quantitative study between different experimental groups (Control, PBS and VEGF) in animals reared in standard conditions (SC-SC) and in enriched environment (SC-EE). Horizontal axes show the ipsilateral and contralateral hemispheres. Vertical axes show number of vessels of the dentate gyrus (a, b) and NeuN positive cells (c, d) per $\mathrm{mm}^{3}$ of the granular cell layer of the dentate gyrus. Mean \pm SEM. ${ }^{* * *} p<0.001$.

showed 6\% $(p=1.000)$ higher density than the non-operated control group. Finally, the VEGF-infused group showed $11 \%(p=0.162)$ higher vascular density than the PBS-infused group (Fig. 2a).

Neuronal density was quantified in the granular cell layer of the dentate gyrus. Although no statistically-significant differences were found, in the ipsilateral hemisphere, the PBS-infused group showed 30\% ( $p=0.131)$ lower neuronal density compared to nonoperated animals. On the other hand, the VEGF-infused group showed $31 \%(p=0.843)$ higher density compared to the PBS-infused group and $8 \%(p=1.000)$ lower than the non-operated group. The observed trend was maintained in the contralateral hemisphere. Whereas the PBS-infused group presented $26 \%$ less vessels than the control group, the VEGF-infused group showed $22 \%$ higher and 9\% lower density than the PBS-infused and non-operated control groups, respectively ( $p=1.000$ for all the experimental groups) (Fig. 2c).

\subsubsection{Enriched environment (SC-EE)}

In animals reared in enriched environment, vascular density showed statistically-significant differences between the studied experimental groups. The PBS-infused group presented $34 \%(p=0.000)$ lower vascular density than the non-operated control group, whereas the VEGF-infused group showed 56\% $(p=0.000)$ higher and $2 \%$ lower $(p=1.000)$ density than the PBS-infused and non-operated control groups, respectively. In the contralateral cortex results were similar. The PBS-infused group showed 33\% $(p=0.000)$ lower density than the control group. On the other hand, the VEGF-infused group presented $52 \%(p=0.000)$ and $1 \%(p=1.000)$ higher vascular density than the PBS-infused and non-operated control groups, respectively (Fig. 2b).

Neuronal density of the granular cell layer showed no statistical differences among the experimental groups although similar results compared to standard reared animals were found. In the ipsilateral cortex, the PBS-infused group presented 26\% $(p=0.513)$ lower neuronal density than the control group, whereas in the VEGF-infused group, the observed neuronal density was $18 \%$ ( $p=0.989)$ higher and $12 \%(p=0.998)$ lower than in the PBS-infused and non-operated control groups, respectively. In the contralateral hemisphere, the PBS-infused and VEGF infused groups showed 13\% and $1 \%$ lower density, respectively, compared to the non-operated control group ( $p=1.000$ in both cases). Finally, the VEGF-infused group presented $14 \%(p=1.000)$ higher neuronal density than the PBS-infused one (Fig. 2d).

Table 2

Effect of enriched environment on vascular and neuronal densities of the dentate gyrus in the ipsilateral (IL) and contralateral (CL) hemispheres of animals reared in standard conditions (SC-SC) and in enriched environment (SC-EE). Average measurements of studied groups (Mean value \pm SEM), and percentage of difference and $p$ value between SC-EE and SC-SC groups of each experimental group (Control, PBS, VEGF).

\begin{tabular}{|c|c|c|c|c|c|c|c|}
\hline & & \multicolumn{2}{|l|}{ Control } & \multicolumn{2}{|l|}{ PBS } & \multicolumn{2}{|l|}{ VEGF } \\
\hline & & SC-SC & SC-EE & SC-SC & SC-EE & SC-SC & SC-EE \\
\hline \multirow[t]{2}{*}{ Vascular density } & IL & $19,307 \pm 726$ & $20,452 \pm 839$ & $18,651 \pm 649$ & $13,352 \pm 750$ & $20,027 \pm 634$ & $20,896 \pm 619$ \\
\hline & $\mathrm{CL}$ & $19,172 \pm 635$ & $19,871 \pm 658$ & $18,238 \pm 564$ & $13,180 \pm 658$ & $20,289 \pm 550$ & $20,030 \pm 537$ \\
\hline \multirow[t]{2}{*}{ Neuronal density } & IL & $148,552 \pm 15,315$ & $209278 \pm 18,471$ & $104,213 \pm 18,471$ & $155,138 \pm 15,818$ & $136,763 \pm 13,061$ & $182,811 \pm 14,439$ \\
\hline & CL & $149,081 \pm 13,363$ & $165,371 \pm 17,563$ & $110,607 \pm 17,563$ & $143,400 \pm 14,127$ & $134,844 \pm 13,025$ & $163,835 \pm 13,363$ \\
\hline
\end{tabular}

\begin{tabular}{|c|c|c|c|c|c|c|c|}
\hline & & \multicolumn{3}{|c|}{ Vascular density (SC-EE vs. SC-SC) } & \multicolumn{3}{|c|}{ Neuronal density (SC-EE vs. SC-SC) } \\
\hline & & Control & PBS & VEGF & Control & PBS & VEGF \\
\hline \multirow[t]{2}{*}{$\%$ Dif } & IL & 5 & -28 & 4 & 41 & 48 & 33 \\
\hline & $\mathrm{CL}$ & 3 & -28 & 1 & 10 & 30 & 22 \\
\hline \multirow[t]{2}{*}{$p$} & $\mathrm{IL}$ & 1.000 & 0.000 & 1.000 & 0.199 & 0.261 & 0.607 \\
\hline & $\mathrm{CL}$ & 1.000 & 0.000 & 1.000 & 1.000 & 1.000 & 1.000 \\
\hline
\end{tabular}




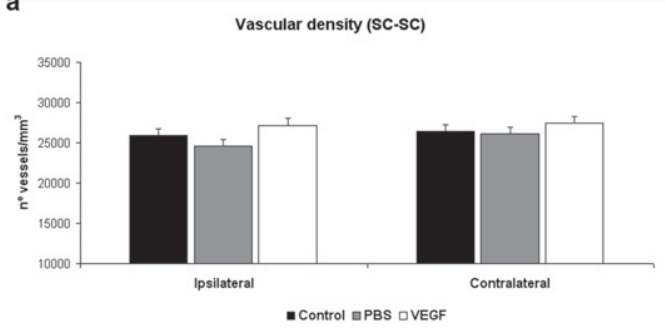

C

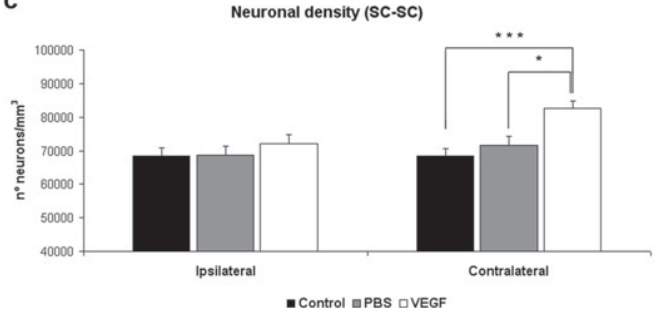

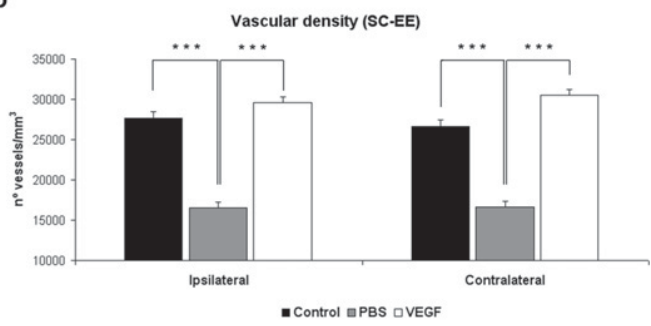

d

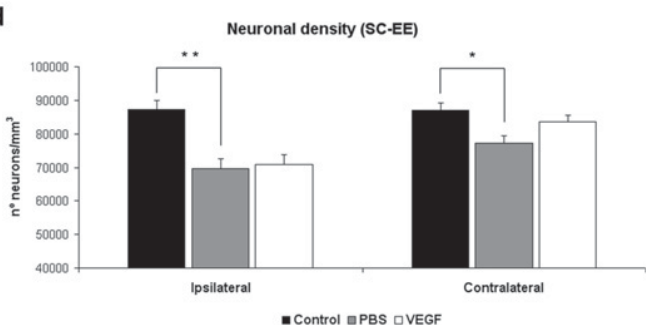

Fig. 3. Effects of VEGF administration on vascular and neuronal densities in the primary visual cortex. Quantitative study between different experimental groups (Control, PBS and VEGF) in animals reared in standard conditions (SC-SC) and in enriched environment (SC-EE). Horizontal axes show the ipsilateral and contralateral hemispheres. Vertical axes show number of vessels ( $\mathrm{a}, \mathrm{b})$ and NeuN positive cells (c, d) per $\mathrm{mm}^{3}$ of the primary visual cortex. Mean \pm SEM. ${ }^{*} p<0.05$; ${ }^{* * *} p<0.001$.

\subsubsection{Enriched environment (SC-EE) vs. standard conditions} $(S C-S C)$

In animals reared in enriched environment, vascular density was $5 \%(p=1.000)$ and $3 \%(p=1.000)$ higher in the non-operated control group (ipsilateral and contralateral hemispheres, respectively). The PBS-infused group presented statistically significant-differences compared to SC-SC reared rats in both hemispheres $(28 \%, p=0.000$ in the ipsilateral cortex; $28 \%, p=0.000$ in the contralateral cortex). In the VEGF-infused group, no effects of enriched environment were found, as SC-EE reared rats showed $4 \%(p=1.000)$ and $1 \%$ $(p=1.000)$ higher vascular density than SC-SC reared rats in the ipsilateral and contralateral cortex, respectively (Table 2).

Although no statistical differences were found in the neuronal density of the granular cell layer in the dentate gyrus, rats raised in SC-EE conditions presented higher neuronal density in all experimental groups. In the ipsilateral hemisphere, 41\%, 48\% and 33\% higher neuronal density was found in SC-EE reared animals in the non-operated controls $(p=0.199)$, the PBS-infused $(p=0.261)$ and the VEGF-infused ( $p=0.607)$ groups, respectively. In the contralateral hemisphere, SC-EE reared rats also showed 10\%, 30\% and $22 \%$ higher neuronal density in the control $(p=1.000)$, PBS $(p=1.000)$ and VEGF-infused $(p=1.000)$ groups (Table 2$)$.

\subsection{Effects of VEGF administration in V1 cortex}

\subsubsection{Standard conditions (SC-SC)}

No significant differences were observed in vascular density in animals reared in standard conditions. In the ipsilateral hemisphere, the PBS-infused group showed 5\% $(p=1.000)$ less vessels than non-operated controls. The VEGF-infused group showed a $5 \%$ $(p=1.000)$ and $10 \%(p=0.464)$ higher vascular density than nonoperated controls and the PBS-infused group. In the contralateral hemisphere, similar results were found. Whereas the PBS-infused group presented $1 \%(p=1.000)$ less vessels than non-operated controls, the VEGF-infused group presented a $4 \%(p=1.000)$ and $5 \%$ $(p=0.998)$ higher vascular density compared to the control and PBS groups, respectively. None of the differences were statistically significant (Fig. 3a).

On the other hand, neuronal density in the ipsilateral hemisphere showed no difference between PBS-infused and nonoperated controls $(0.38 \% ; p=0.977)$. The VEGF-infused group presented 5\% higher neuronal density than both the control and PBS groups ( $p=0,998$ and $p=1.000$ respectively). Nonetheless, the VEGF group showed statistically-significant more neurons than the non-operated controls $(20 \% ; p=0.000)$ and the PBS-infused $(15 \%$; $p=0.030$ ) group in the contralateral hemisphere. The PBS-infused group also showed $5 \%$ more neurons than controls $(p=1.000)$ (Fig. 3c).

\subsubsection{Enriched environment (SC-EE)}

In animals reared in enriched environment after minipump implantation, significant differences were found in the vascular density of the primary visual cortex. In the ipsilateral hemisphere, the PBS-infused group showed $40 \%(p=0.000)$ lower vascular density than non-operated controls and $79 \%(p=0.000)$ lower than the VEGF-infused group. Thus, $7 \%$ higher density was observed in the VEGF-infused group compared to the non-operated control group, resulting in a statistically-insignificant difference. Similar results were found in the contralateral hemisphere. Whereas the PBSinfused group showed $37 \%(p=0.000)$ lower vascular density than non-operated controls, the VEGF-infused group showed a significantly higher density than both the control $(15 \% ; p=0.014)$ and PBS-infused groups ( $83 \% ; p=0.000$ ) (Fig. 3b).

In the ipsilateral cortex, lower neuronal density compared to enriched-environment reared controls was found in both the PBS and VEGF-infused groups, $(20 \%, p=0.001$ and $19 \%, p=0.074$ respectively). Therefore, the PBS and VEGF-infused groups showed similar neuronal density to each other $(2 \% ; p=1.000)$. In the contralateral hemisphere, the PBS-infused group also showed a statisticallysignificant lower neuronal density than non-operated controls $(11 \% ; p=0.044)$. On the contrary, the VEGF-infused group presented 3\% less neurons than the control group, resulting in a non-significant difference $(p=1.000)$. Finally, the VEGF-infused group showed $8 \%(p=0.477)$ higher density than the PBS-infused group (Fig. 3d).

\subsubsection{Enriched environment (SC-EE) vs. standard conditions (SC-SC)}

In the vascular density of non-operated controls, no statistical differences were found in rats reared in enriched environment compared to rats reared in standard conditions $(p=1.000$ in both hemispheres). In the VEGF-infused group, SC-EE reared rats also presented 9\% (ipsilateral; $p=0.564$ ) and 10\% (contralateral; 
Table 3

Effect of enriched environment on vascular and neuronal densities of the primary visual cortex in the ipsilateral (IL) and contralateral (CL) hemispheres of animals reared in standard conditions (SC-SC) and in enriched environment (SC-EE). Average measurements of studied groups (Mean value \pm SEM), and percentage of difference and $p$ value between SC-EE and SC-SC groups of each experimental group (Control, PBS, VEGF).

\begin{tabular}{|c|c|c|c|c|c|c|c|c|}
\hline & & & \multicolumn{2}{|l|}{ Control } & \multicolumn{2}{|l|}{ PBS } & \multicolumn{2}{|l|}{ VEGF } \\
\hline & & & SC-SC & SC-EE & SC-SC & SC-EE & SC-SC & SC-EE \\
\hline \multirow{6}{*}{$\begin{array}{l}\text { Vascular } \\
\text { density } \\
\text { Neuronal } \\
\text { density }\end{array}$} & IL & & $25,940 \pm 798$ & $27,677 \pm 798$ & $24,600 \pm 788$ & $16,496 \pm 724$ & $27,153 \pm 872$ & $29,572 \pm 758$ \\
\hline & $\mathrm{CL}$ & & $26,432 \pm 794$ & $26,591 \pm 850$ & $26,124 \pm 863$ & $16,623 \pm 765$ & $27,404 \pm 850$ & $30,497 \pm 716$ \\
\hline & IL & & $68,281 \pm 2465$ & $87,284 \pm 2662$ & $68,547 \pm 2772$ & $69,523 \pm 3170$ & $71,966 \pm 2732$ & $70,787 \pm 2903$ \\
\hline & CL & & $68,505 \pm 2111$ & $86,998 \pm 2369$ & $71,650 \pm 2629$ & $77,157 \pm 2338$ & $82,571 \pm 2297$ & $83,573 \pm 2102$ \\
\hline & & & & \multicolumn{2}{|c|}{ Vascular density (SC-EE vs. SC-SC) } & \multicolumn{3}{|c|}{ Neuronal density (SC-EE vs. SC-SC) } \\
\hline & & & Control & PBS & VEGF & Control & PBS & VEGF \\
\hline \multirow[t]{2}{*}{$\%$ Dif } & & IL & 2 & -33 & 9 & 28 & 1 & -1 \\
\hline & & $\mathrm{CL}$ & 0.006 & -36 & 10 & 27 & 7 & 1 \\
\hline \multirow[t]{2}{*}{$p$} & & IL & 1.000 & 0.000 & 0.564 & 0.001 & 1.000 & 1.000 \\
\hline & & $\mathrm{CL}$ & 1.000 & 0.000 & 0.179 & 0.000 & 1.000 & 1.000 \\
\hline
\end{tabular}

$p=0.179$ ) higher vascular density. In contrast, in the PBS-infused group SC-EE reared rats showed statistically lower vascular density compared to SC-SC reared rats in both hemispheres (33\% in the ipsilateral hemisphere, $p=0.000 ; 36 \%$ in the contralateral hemisphere, $p=0.000$ ) (Table 3).

Neuronal density of non-operated controls presented a statistically-significant increase in rats reared in SC-EE conditions ( $28 \%$ in the ipsilateral hemisphere, $p=0.001 ; 27 \%$ in the contralateral hemisphere, $p=0.000$ ). No effects of enriched environment were found in the PBS and VEGF-infused groups. In the PBSinfused group, SC-EE reared rats showed $1 \%(\mathrm{p}=1.000)$ and $7 \%$ $(p=1.000)$ higher neuronal density compared to SC-SC reared rats in the ipsilateral and contralateral hemispheres respectively. The VEGF-infused group presented $1 \%$ lower $(p=1.000)$ and $1 \%$ higher $(p=1.000)$ neuronal density in rats reared in an enriched environment, this being a statistically-insignificant difference (Table 3).

\subsection{VEGF co-expression in DG}

VEGF expression was similar in the dentate gyrus of all experimental groups and most of the VEGF-positive cells co-localized with GFAP (Fig. 4a-c). VEGF/NeuN immunofluorescence did not show differences among the experimental groups, although some VEGF-positive neurons were found (Fig. 4d-f). No co-localization with LEA was observed (Fig. 4g-i). Therefore, VEGF expression corresponds to astrocytes in the dentate gyrus at P46.

\subsection{VEGF co-expression in V1 cortex}

Double immunofluorescence of VEGF/GFAP showed a significantly lower expression of both antigens in non-operated rats compared to the PBS and VEGF groups, higher expression being observed in the latter (Fig. 5a-c). Most of the VEGF-positive cells corresponded to astrocytes, as they co-localized with GFAP. In addition, VEGF/NeuN immunolabelling confirmed that co-localization with NeuN positive cells is lower in both infused groups (PBS and VEGF groups) compared to the control group (Fig. 5d-f). No colocalization with LEA was observed in any of the experimental groups (Fig. 5g-i). Thus, the majority of VEGF expression corresponds to astrocytes in the primary visual cortex at P46.

\section{Discussion}

\subsection{Effects of VEGF administration}

VEGF administration during the critical period of the visual cortex shows different responses depending on the rearing environment. Angiogenic, neuroprotective and neurogenic properties have been described for VEGF in the brain, as well as its influence over hippocampus-dependent memory $[12,14,15]$.

Our results revealed an improved trend during the learning process induced by VEGF administration compared to non-operated and PBS-infused animals raised in standard conditions although no beneficial effects were found in long-term spatial memory and reversal learning. Since the dentate gyrus is the primary afferent into the hippocampus, we carried out a quantitative analysis of vascular and neuronal densities of DG. Results did not show statistically-significant differences in vascular and neuronal densities of the dentate gyrus in the studied experimental groups reared in standard conditions. Nevertheless, in the VEGF-infused group, an increase in neuronal density of the granular cell layer of DG was observed. Recently, it has been shown that VEGF promotes hippocampus-dependent memory, increasing the synaptic strength, independently of its effects on neurogenesis and angiogenesis [16], which could explain the lack of neurovascular changes found in the dentate gyrus.

In animals reared in enriched environment, data also showed a significant improvement during learning acquisition in the VEGFinfused group compared to the PBS-infused and non-operated control groups. Moreover, whereas PBS-infusion impaired longterm spatial memory, VEGF administration counteracted this negative effect and showed an improved reversal test as well. It has been previously described that VEGF improves cognition in rodents and our data revealed similar results when administered intracortically in developing rats. Furthermore, quantitative results showed that PBS-infusion induced a negative effect on vascular density in the dentate gyrus, which was reverted with VEGF administration in enriched animals. Consistent with previous studies that demonstrated that angiogenesis was critical to normal learning and memory [32], our data also suggest that the vascular component of DG could be determinant, as increased vascular density was found in the VEGF-infused group. VEGF can activate neurogenesis by targeting neurons directly or protecting them indirectly through its angiogenic effect $[14,33]$. Nonetheless, in addition to augmenting the number of newborn cells in the brain, newly formed vessels are needed for their metabolic and trophic demands [34]. Thus, several studies have indicated that neurogenesis is not sufficient by itself for memory enhancement $[16,35,36]$. Our results suggest that vascularization is related to normal learning although VEGF could also increase connectivity between mature neurons to enhance learning as well.

On the other hand, the absence of VEGF effects in animals reared in standard conditions but not in enriched ones could be due to enhanced experience after minipump implantation, as these animals were reared in an enriched environment only after the surgery. Interaction of experience with time sensitive 
Control
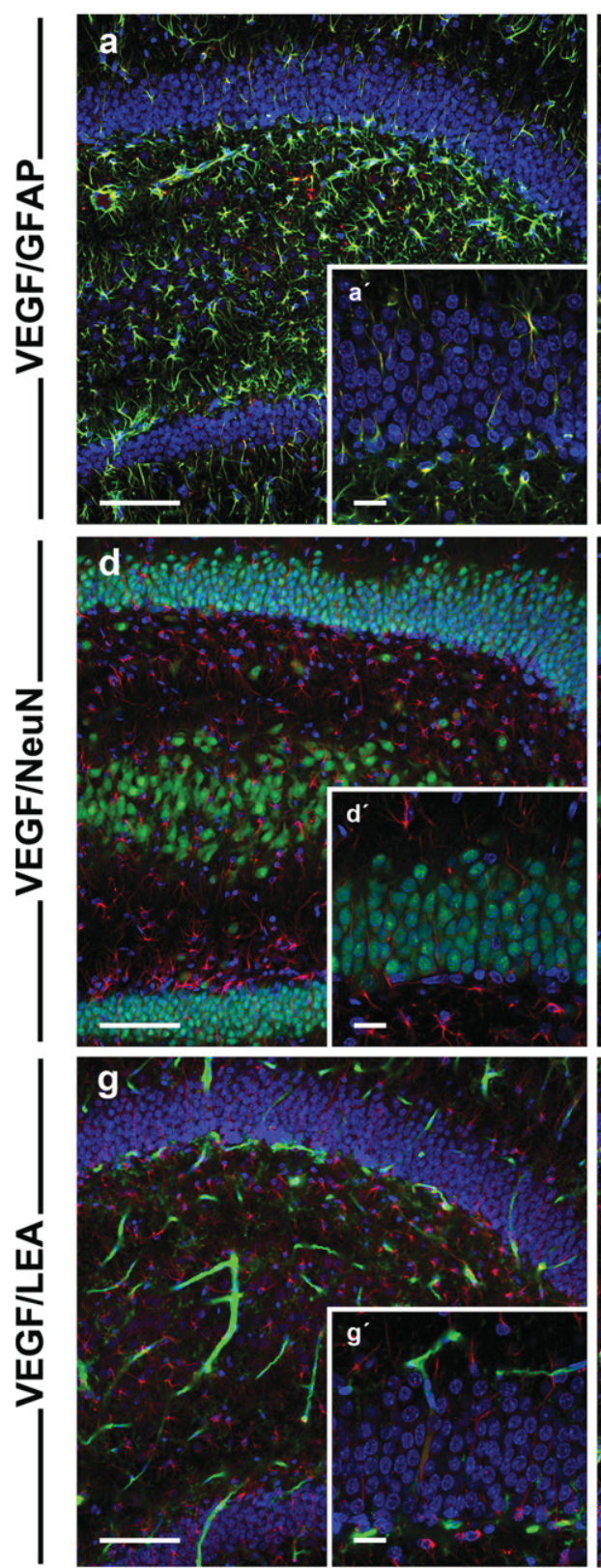

PBS

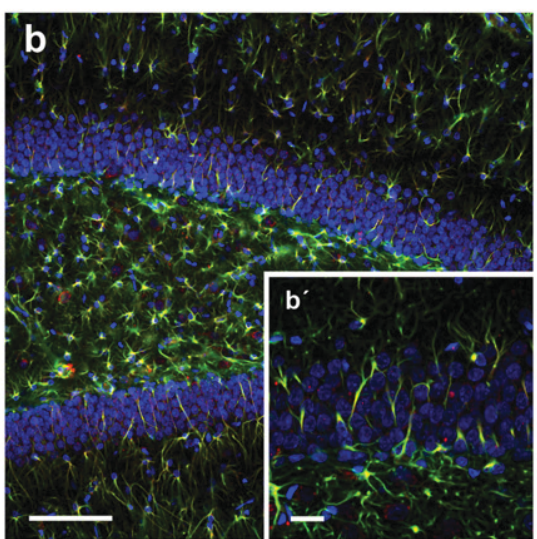

e
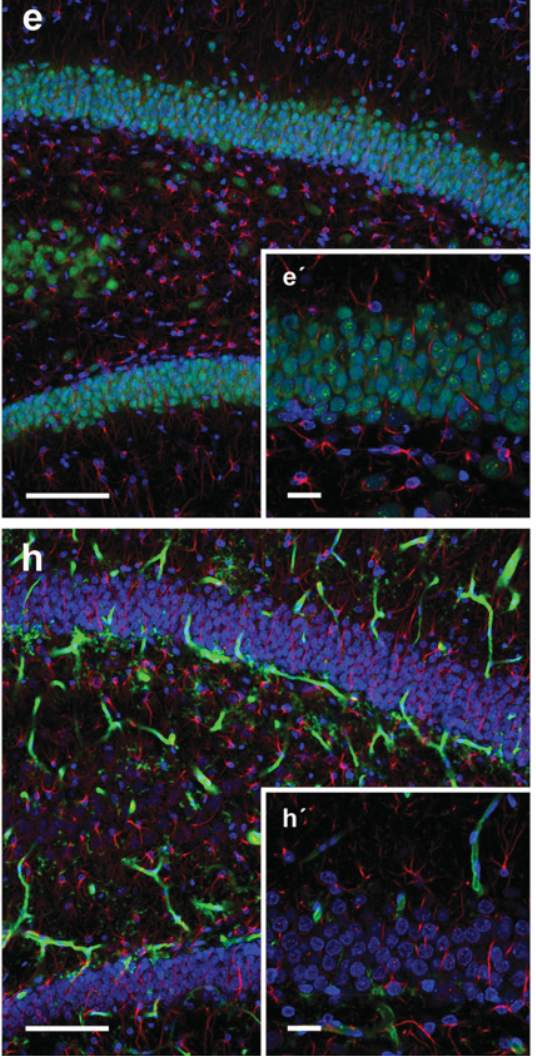

VEGF
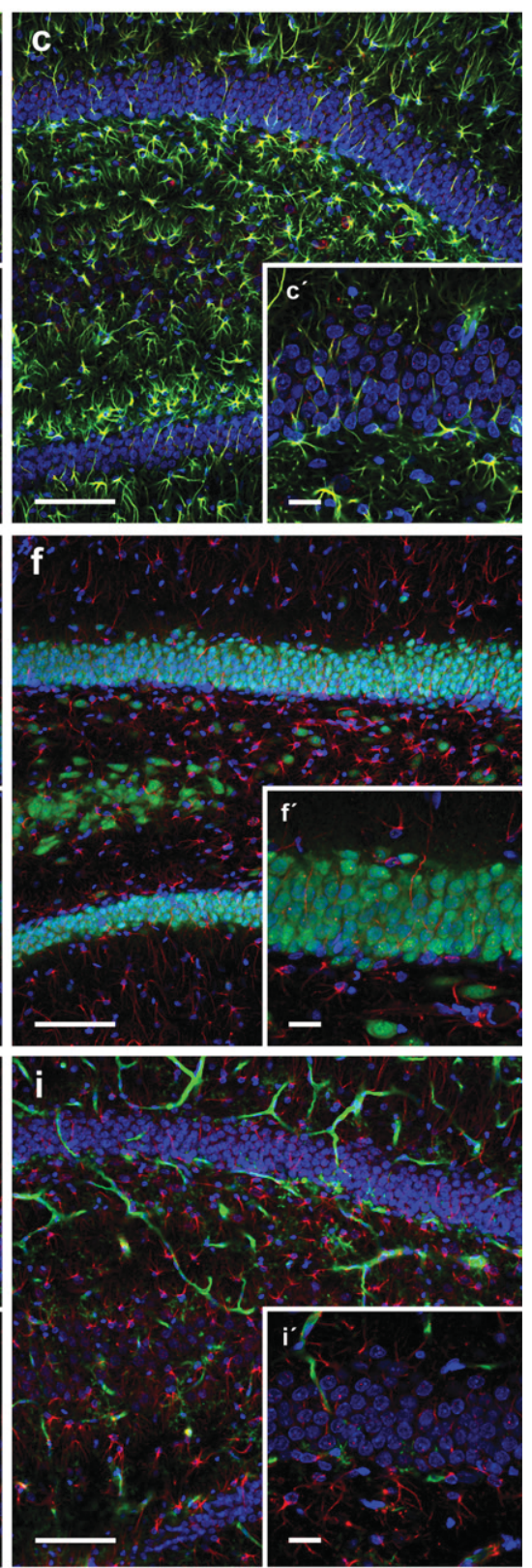

Fig. 4. VEGF/GFAP, VEGF/NeuN and VEGF/LEA co-expression in the dentate gyrus. Confocal images of double immunolabelling of VEGF and GFAP (a-c), VEGF and NeuN (d-f), and VEGF and LEA ( $\mathrm{g}-\mathrm{i})$ to determine if VEGF positivity corresponds to astrocytes, neurons or endothelial cells respectively. Blue corresponds to Hoeschst 33258 positive nuclei. Scale bar $=100 \mu \mathrm{m}(\mathrm{a}-\mathrm{i})$ and $20 \mu \mathrm{m}\left(\mathrm{a}^{\prime}-\mathrm{i}^{\prime}\right)$.

injury-induced changes has been described as shaping brain reorganization in a long-lasting manner, including areas proximal to the injury that might be particularly important for functional outcome [37]. Therefore, our results suggest that VEGF infusion might aid recovery from minipump implantation-induced-lesions.

\subsection{DG-V1 cortex connection}

Whereas most studies to determine VEGF function in learning and memory used intracerebroventricular $[8,38,39]$ or intrahippocampal administration [40], in our work, VEGF has been administered immediately anterior to the primary visual cortex and a direct arrival to the DG could be discarded. The visual cortex provides a crucial sensory input to the hippocampus and is a key component for the creation of visuospatial memories [41]. Diverse studies have suggested that experience-dependent synaptic plasticity occurs in the adult primary visual cortex, which could constitute a key element in neocortico-hippocampal transfer of information $[41,42]$. This relationship between learning and visual cortical changes has also been observed in rodents $[43,44]$. In addition to the hippocampus and the parahippocampal region, the enthorinal cortex [45] and postrhinal cortex [46] also have a reciprocal connection with the visual system. On the other hand, the retrosplenial cortex has also been demonstrated to contribute to memory and navigation, and being anatomically close to V1, a direct effect of VEGF should not be discarded [47].

Our data demonstrate that effects of VEGF administration in the DG are also present in the visual cortex, near to the cannula 

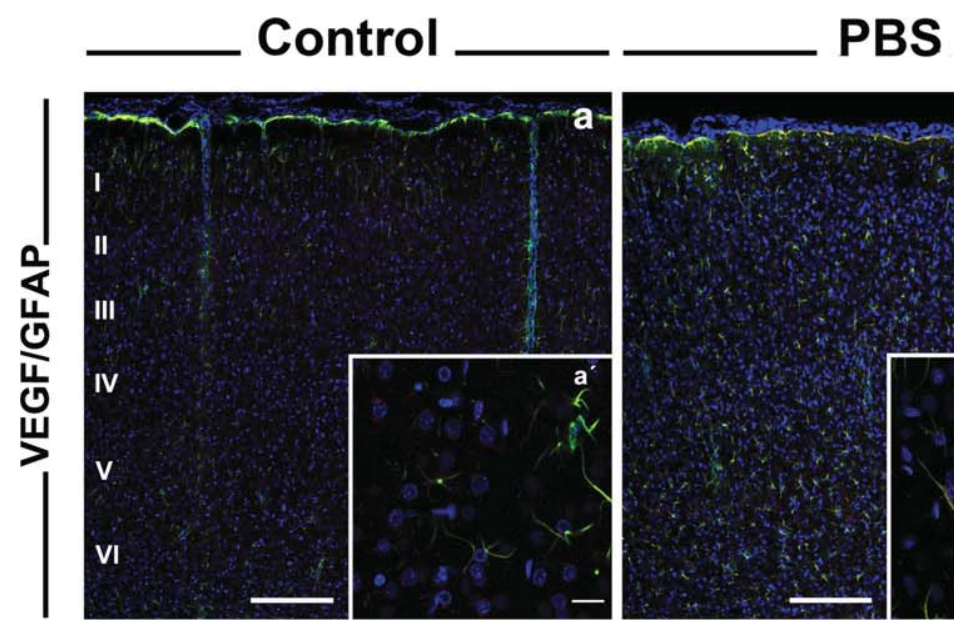

VEGF
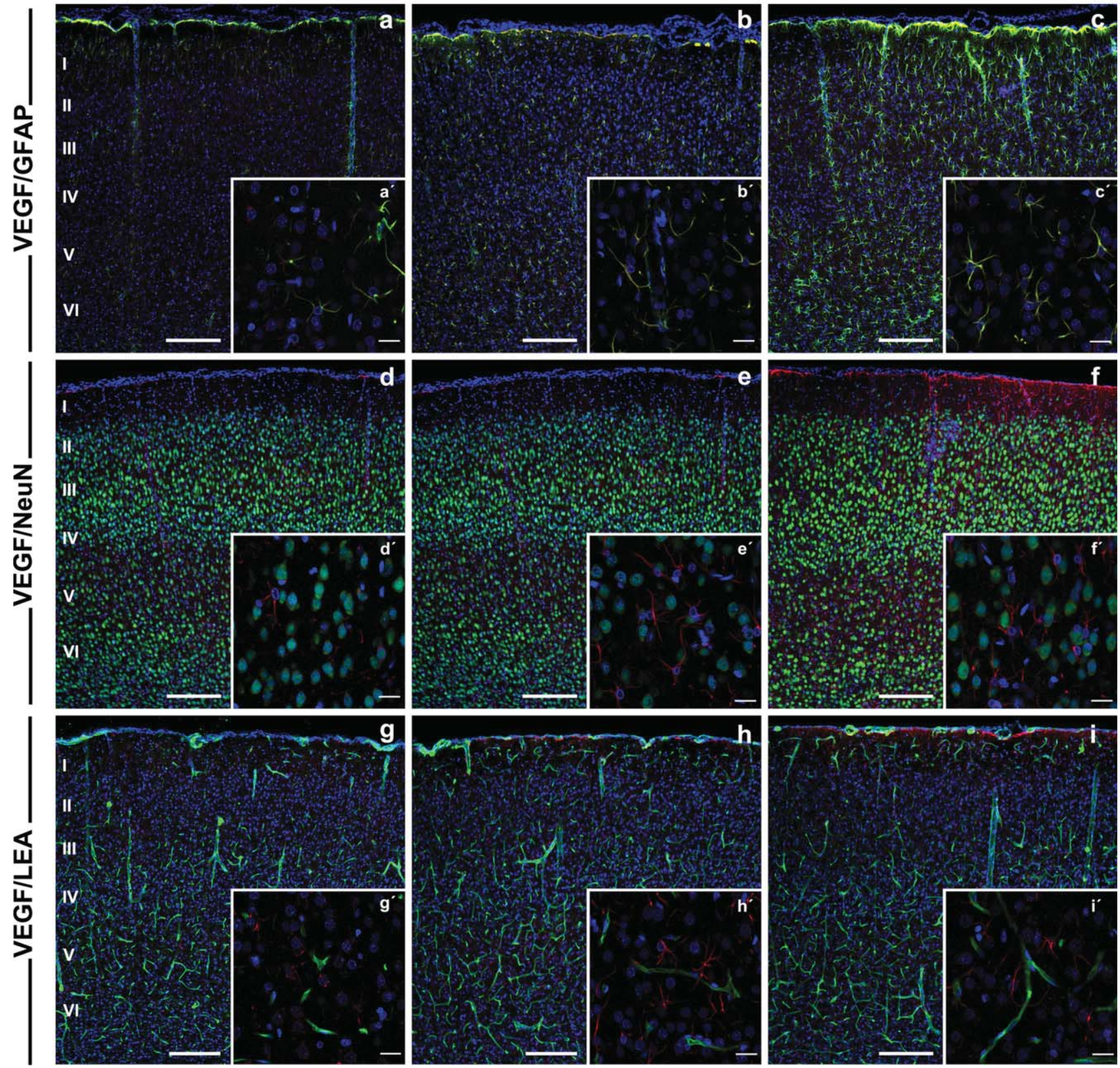

Fig. 5. VEGF/GFAP, VEGF/NeuN and VEGF/LEA co-expression in the primary visual cortex. Confocal images of double immunolabelling of VEGF and GFAP (a-c), VEGF and NeuN (d-f), and VEGF and LEA (g-i) to determine if VEGF positivity corresponds to astrocytes, neurons or endothelial cells respectively. Blue corresponds to Hoeschst 33258 positive nuclei. Scale bar $=200 \mu \mathrm{m}(\mathrm{a}-\mathrm{i})$ and $20 \mu \mathrm{m}\left(\mathrm{a}^{\prime}-\mathrm{i}^{\prime}\right)$.

placement. As in the dentate gyrus, no effect of VEGF administration was observed in the neuronal and vascular densities of the primary visual cortex in standard conditions. Previous studies in our group demonstrated that minipump implantation produced negative effects in the V1 cortex that were reverted after 1 week of VEGF administration $[48,49]$. In this case, we carried out a chronic VEGF or vehicle administration and therefore a chronic minipump implantation during four weeks. This time lapse is enough to repair the surrounding tissue, thus recovering from injuries induced by minipump implantation. In addition, focal small lesions such as the one produced by cannula implantation can be repaired easily [50].

In contrast, in animals reared in enriched environment, the PBSinfused group showed a significantly lower vascular density, similar to that observed in DG. VEGF infusion counteracted this negative effect on microvascularization and reached non-operated control values. The PBS-infusion group also presented a significantly lower neuronal density compared to non-operated controls. VEGF administration did not revert to the neuronal density in the primary visual cortex as both the PBS and VEGF-infused groups presented values similar to the standard-reared control group. It has been proposed that there is a ceiling effect of VEGF and FGF-2 for inducing changes on neurovascular components after injury [39]. This fact could explain that VEGF acts only if the studied parameters are lower than physiological values.

Therefore, the connections described above could explain the fact that changes in DG are reflected in the primary visual cortex and vice versa. 


\subsection{Enriched environment vs. standard conditions}

In addition to VEGF administration, an enriched environment by itself presents direct effects on studied neurovascular components and on the learning process. Exposure to an enriched environment has been shown to induce morphological changes in the brain, both in normal and pathological conditions [51,52]. These morphological changes include structural reorganization, especially in the visual cortex [53], which is associated with improved learning and memory and enhanced neural plasticity [54].

As has been observed before in the adult brain [53,54], our results have shown that enrichment exposure until P46 in the nonoperated control group enhances the learning process and reversal learning compared to standard-reared rats. Enrichment-induced improvement of learning has been related to increased synaptic plasticity or hippocampal neurogenesis, among other effects $[9,55,56]$. Even if no differences in vascular density were observed, our results showed that enrichment induced an enhancement in the granular cell layer of the dentate gyrus. Although it has been reported that enrichment induces a survival-promoting effect in the dentate gyrus [9], the PBS and VEGF-infused groups did not show any improvement during the learning process. Nonetheless, the enriched environment enhances reversal learning in VEGF-infused animals whereas in PBS-infused ones, it impairs the performance. In fact, recent studies have suggested that neurogenesis is not the unique supporter of the enhancement induced by enrichment [35,36] since newly proliferated neurons need 3-4 weeks to be functionally incorporated into existing learning networks $[57,58]$. In agreement with these studies, our results showed that in the VEGF and PBS-infused groups, rearing in enriched environment also induced an increase in neuronal density of the granular cell layer in the dentate gyrus that was not accompanied by an improvement during the learning process.

On the other hand, previous studies have demonstrated that rearing in an enriched environment from birth to adulthood increases vascular density in the rat visual cortex, and that this is correlated with an increase in VEGF expression [25]. In addition, it was observed that exposure to EE from P18 onwards increased microvasculature at P25 [48]. Nevertheless, rearing in EE during the entire critical period (from P18 to P46) induced an increase in neuronal density. Enrichment elicits neuroprotective responses as it induces changes in genes related to neuroprotection [59], and expression of angioglioneurins such as BDNF [60] and VEGF [25] are increased as well. Therefore, enrichment elicits neurorescuer effects if applied throughout the critical period. In contrast, no beneficial effects were found in the V1 of the PBS and VEGF-infused groups, probably due to the minipump implantationinduced lesion. Enrichment-induced effects are different when applied in normal or pathological situations [61-63].

\subsection{VEGF co-expression in V1 and DG}

In the developing brain, VEGF is initially produced by neurons. At P13 neuronal expression of VEGF starts to diminish and astrocytic expression becomes more evident until localization of VEGF switches from being predominantly neuronal to glial at P24 [25]. However, in the hypoxic brain, high levels of neuronal and glial VEGF are maintained until P33 [63]. Our results showed that VEGF immunonoreactivity co-localized with astrocytes and neurons in the V1 cortex although most VEGF-positive cells corresponded to astrocytes in all experimental groups.

On the other hand, whereas our results showed that VEGF is expressed in astrocytes and neurons of the granular cell layer and the polymorphic layer of the dentate gyrus, as has been previously reported [12], no co-localization with endothelial cells has been found. Moreover, most VEGF expression corresponds to astrocytes.
Some authors have proposed that astrocytes are an important niche for neurogenesis [64], and that astrocytes-derived VEGF expression could induce an increase in angiogenesis as they are localized adjacent to cerebral vessels [12]. Neuronal expression of VEGF has also been proposed to play an important role in the regulation of neurogenesis [12].

\section{Conclusions}

VEGF infusion as well as enriched environment induces neurovascular and cognitive effects in developing rats. VEGF administration produces an enhancement during the learning process and acts as an angiogenic factor in order to counteract minipump implantation-induced damage, revealing that DG vascularization is critical for normal learning. On the other hand, enriched environment acts on the neuronal component of DG and V1 cortex. Moreover, results showed learning enhancement only in non-operated rats, leading to the conclusion that in addition to neurogenesis, vascularization plays a pivotal role for learning and memory.

\section{Acknowledgements}

This study has been supported by SAIOTEK (Industry Dept., Government of the Basque Country), IT/491/10 (Basque Government) and UFI 11/32 (UPV/EHU). N. Ortuzar is supported by a predoctoral grant (FPU) from the Spanish Ministry of Education and a postdoctoral grant of the University of the Basque Country (UPV/EHU). Confocal microscopy analysis was performed at the "Servicio de Microscopia Analítica y de Alta Resolución en Biomedicina" (High Resolution Analytical Microscopy Services for Biomedicine) at the University of the Basque Country (UPV/EHU).

\section{Appendix A. Supplementary data}

Supplementary data associated with this article can be found, in the online version

\section{References}

[1] Zacchigna S, Lambrechts D, Carmeliet P. Neurovascular signalling defects in neurodegeneration. Nature Reviews Neuroscience 2008;9:169-81.

[2] Argandoña EG, Bengoetxea H, Ortuzar N, Bulnes S, Rico-Barrio I, Lafuente JV. Vascular Endothelial Growth Factor: Adaptative changes in the Neurogliovascular unit. Current Neurovascular Research 2012;9:72-81.

[3] Ferrara N, Gerber HP, LeCouter J. The biology of VEGF and its receptors. Nature Medicine 2003;9:669-76.

[4] Jin K, Zhu Y, Sun Y, Mao XO, Xie L, Greenberg DA. Vascular endothelial growth factor (VEGF) stimulates neurogenesis in vitro and in vivo. Proceedings of the National Academy of Sciences of the United States of America 2002;99:11946-50.

[5] Rosenstein JM, Krum JM. New roles for VEGF in nervous tissue - beyond blood vessels. Experimental Neurology 2004:187:246-53.

[6] Storkebaum E, Lambrechts D, Carmeliet P. VEGF: once regarded as a specific angiogenic factor, now implicated in neuroprotection. BioEssays 2004;26:943-54.

[7] Kaya D, Gürsoy-Ozdemir Y, Yemisci M, Tuncer N, Aktan S, Dalkara T. VEGF protects brain against focal ischemia without increasing blood-brain permeability when administered intracerebroventricularly. Journal of Cerebral Blood Flow and Metabolism 2005;25:1111-8.

[8] Sun Y, Jin K, Childs JT, Xie L, Mao XL, Greenberg DA. Vascular endothelial growth factor-B (VEGFB) stimulates neurogenesis: evidence from knockout mice and growth factor administration. Developmental Biology 2006;289:329-35.

[9] Kempermann G, Kuhn HG, Gage FH. More hippocampal neurons in adult mice living in an enriched environment. Nature 1997;386:493-5.

[10] Louissaint Jr A, Rao S, Leventhal C, Goldman SA. Coordinated interaction of neurogenesis and angiogenesis in the adult songbird brain. Neuron 2002;34:945-60.

[11] Greenberg DA, Jin K. From angiogenesis to neuropathology. Nature 2005;438:954-9.

[12] Cao L, Jiao X, Zuzga DS, Liu Y, Fong DM, Young D, et al. VEGF links hippocampal activity with neurogenesis, learning and memory. Nature Genetics 2004;36:827-35. 
[13] During MJ, Cao L. VEGF, a mediator of the effect of experience on hippocampal neurogenesis. Current Alzheimer Research 2006;3:29-33.

[14] Plaschke K, Staub J, Ernst E, Marti HH. VEGF overexpression improves mice cognitive abilities after unilateral common carotid artery occlusion. Experimental Neurology 2008;214:285-92.

[15] Kim BW, Choi M, Kim YS, Park H, Lee HR, Yun CO, et al. Vascular endothelia growth factor (VEGF) signalling regulates hippocampal neurons by elevation of intracellular calcium and activation of calcium/calmodulin protein kinase II and mammalian target of rapamycin. Cellular Signalling 2008;20: 714-25.

[16] Licht T, Goshen I, Avital A, Kreisel T, Zubedat S, Eavri R, et al. Reversible modulations of neuronal plasticity by VEGF. Proceedings of the National Academy of Sciences of the United States of America 2011;108:5081-6.

[17] Rosenzweig MR, Bennet EL, Hebert M, Morimoto H. Social grouping cannot account for cerebral effects of enriched environments. Brain Research 1978;153:563-76.

[18] Nithianantharajah J, Hannan AJ. The neurobiology of brain and cognitive reserve: Mental and physical activity as modulators of brain disorders. Progress in Neurobiology 2009;89:369-82.

[19] Martínez-Cué C, Rueda N, García E, Davisson MT, Schmidt C, Flórez J. Behavioral, cognitive and biochemical responses to different environmental conditions in male Ts65Dn mice, a model of Down syndrome. Behavioural Brain Research 2005;163:174-85.

[20] Bennett EL, Rosenzweig MR, Diamond MC. Rat brain: effects of environmental enrichment on wet and dry weights. Science 1969;163:825-6.

[21] Diamond MC, Ingham CA, Johnson RE, Bennett EL, Rosenzweig MR. Effects of environmental morphology of rat cerebral cortex and hippocampus. Journal of Neurobiology 1976;7:75-86.

[22] Greenough WT, Volkmar FR. Pattern of dendritic branching in occipital cortex of rats reared in complex environments. Experimental Neurology 1973;40:491-504.

[23] Rosenzweig MR, Bennett EL, Diamond MC, Wu SY, Slagle RW, Saffran E. Influences of environmental complexity and visual stimulation on development of occipital cortex in rat. Brain Research 1969;14:427-45.

[24] Faherty CJ, Kerley D, Smeyne RJ. A Golgi-Cox morphological analysis of neuronal changes induced by environmental enrichment. Brain Research Developmental Brain Research 2003;141:55-61.

[25] Bengoetxea H, Argandoña EG, Lafuente JV. Effects of visual experience on vascular endothelial growth factor expression during the postnatal development of the rat visual cortex. Cerebral Cortex 2008;18:1630-9.

[26] Ickes BR, Pham TM, Sanders LA, Albeck DS, Mohammed AH, Granholm AC. Longterm environmental enrichment leads to regional increases in neurotrophin levels in rat brain. Experimental Neurology 2000;164:45-52.

[27] Pham TM, Winblad B, Granholm AC, Mohammed AH. Environmental influences on brain neurotrophins in rats. Pharmacology Biochemistry and Behavior 2002;73:167-75.

[28] Cancedda L, Putignano E, Sale A, Viegi A, Berardi N, Maffei L. Acceleration of visual system development by environmental enrichment. Journal of Neuroscience 2004;24:4840-8.

[29] Hooks BM, Chen C. Critical periods in the visual system: changing views for a model of experience-dependent plasticity. Neuron 2007;56:312-26.

[30] Fagiolini M, Pizzorusso T, Berardi N, Domenici L, Maffei L. Functional postnatal development of the rat primary visual cortex and the role of visual experience: dark rearing and monocular deprivation. Vision Research 1994;34: 709-20.

[31] Fagiolini M, Hensch TK. Inhibitory threshold for critical-period activation in primary visual cortex. Nature 2000;404:183-6.

[32] Kerr AL, Steuer EL, Pochtarev V, Swain RA. Angiogenesis but not neurogenesis is critical for normal learning and memory acquisition. Neuroscience 2010;171:214-26.

[33] Marti HJ, Bernaudin M, Bellail A, Schoch H, Euler M, Petit E, et al Hypoxia-induced vascular endothelial growth factor expression precedes neovascularization after cerebral ischemia. American Journal of Pathology 2000;156:965-76.

[34] Palmer TD, Willhoite AR, Gage FH. Vascular niche for adult hippocampal neurogenesis. Journal of Comparative Neurology 2000;425:479-94.

[35] Meshi D, Drew MR, Saxe M, Ansorge MS, David D, Santarelli L, et al. Hippocampal neurogenesis is not required for behavioural effects of environmental enrichment. Nature Neuroscience 2006;9:729-31.

[36] Shors TJ, Townsend DA, Zhao M, Kozorovitskiy Y, Gould E. Neurogenesis may relate to some but not all types of hippocampal-dependent learning. Hippocampus 2002;12:578-84.

[37] Jones TA, Jefferson SC. Reflections of experience-expectant development in repair on the adult damaged brain. Developmental Psychobiology 2011;53:466-75.
[38] Wang Y, Galvan V, Gorostiza O, Ataie M, Jin K, Greenberg DA. Vascular endothelial growth factor improves recovery of sensorimotor and cognitive deficits after focal cerebral ischemia in the rat. Brain Research 2006;1115:186-93.

[39] Thau-Zuchman O, Shohami E, Alexandrovich AG, Leker RR. Combination of vascular endothelial and fibroblast growth factor 2 for induction of neurogenesis and angiogenesis after traumatic brain injury. Journal of Molecular Neuroscience 2012;47:166-72.

[40] Pati S, Orsi SA, Moore AN, Dash PK. Intra-hippocampal administration of the VEGF receptor blocker PTK787/ZK222584 impairs long-term memory. Brain Research 2009;1256:85-91.

[41] Tsanov M, Manahan-Vaughan D. Synaptic plasticity from visual cortex to hippocampus: systems integration in spatial information processing. Neuroscientist 2008;14:584-97.

[42] Heynen AJ, Bear MF. Long-term potentiation of thalamocortical transmission in the adult visual cortex in vivo. Journal of Neuroscience 2001;21:9801-13.

[43] Frenkel MY, Sawtell NB, Diogo AC, Yoon B, Neve RL, Bear MF. Instructive effect of visual experience in mouse visual cortex. Neuron 2006;51:339-49.

[44] Karmarkar UR, Dan Y. Experience-dependent plasticity in adult visual cortex. Neuron 2006;52:577-85.

[45] Burwell RD, Amaral DG. Cortical afferents of the perirhinal, postrhinal, and entorhinal cortices of the rat. Journal of Comparative Neurology 1998;398:179-205.

[46] Brown MV, Aggleton JP. Recognition memory: what are the roles of the perirhinal cortex and hippocampus. Nature Reviews Neuroscience 2001;2:51-61.

[47] Vann SD, Aggleton JP, Maguire EA. What does the retrosplenial cortex do? Nature Reviews Neuroscience 2009;10:792-802.

[48] Ortuzar N, Argandoña EG, Bengoetxea H, Lafuente JV. Combination of intracortically administered VEGF and environmental enrichment enhances brain protection in developing rats. Journal of Neural Transmission 2011;118:135-44.

[49] Argandoña EG, Bengoetxea H, Bulnes S, Rico-Barrio I, Ortuzar N, Lafuente JV. Effect of intracortical vascular endothelial growth factor infusion and blockade during the critical period in the rat visual cortex. Brain Research 2012;1473:141-54

[50] Ballantyne AO, Spilkin AM, Hesselink J, Trauner DA. Plasticity in the developing brain: intellectual, language and academic functions in children with ischaemic perinatal stroke. Brain 2008;131:2975-85.

[51] Dahlqvist P, Zhao L, Johansson IM, Mattsson B, Johansson BB, Seckl JR, et al. Environmental enrichment alters nerve growth factor-induced gene $A$ and glucocorticoid receptor messenger RNA expression after middle cerebral artery occlusion in rats. Neuroscience 1999;93:527-35.

[52] Johansson BB, Belichenko PV. Neuronal plasticity and dendritic spines: effect of environmental enrichment on intact and postischemic rat brain. Journal of Cerebral Blood Flow and Metabolism 2002;22:89-96.

[53] Rampon C, Tang YP, Goodhouse J, Shimizu E, Kyin M, Tsien JZ. Enrichment induces structural changes and recovery from nonspatial memory deficits in CA1 NMDAR1-knockout mice. Nature Neuroscience 2000;3:238-44.

[54] van Praag H, Kempermann G, Gage FH. Neural consequences of environmental enrichment. Nature Reviews Neuroscience 2000;1:191-8.

[55] Nithianantharajah J, Hannan AJ. Enriched environments, experiencedependent plasticity and disorders of the nervous system. Nature Reviews Neuroscience 2006;7:697-709.

[56] Pizzorusso T, Berardi N, Maffei L. A richness that cures. Neuron 2007;54:508-10.

[57] Overstreet LS, Hentges ST, Bumaschny VF, de Souza FS, Smart JL, Santangelo $\mathrm{AM}$, et al. A transgenic marker for newly born granule cells in dentate gyrus. Journal of Neuroscience 2004;24:3251-9.

[58] Kee N, Teixeira CM, Wang AH, Frankland PW. Preferential incorporation of adult-generated granule cells into spatial memory networks in the dentate gyrus. Nature Neuroscience 2007;10:355-62.

[59] Paban V, Chambon C, Manrique C, Touzet C, Alescio-Lautier B. Neurotrophic signalling molecules associated with cholinergic damage in young and aged rats: environmental enrichment as potential therapeutic agent. Neurobiology of Aging 2011;32:470-85.

[60] Sale A, Maya Vetencourt JF, Medini P, Cenni MC, Baroncelli L, De Pasquale $\mathrm{R}$, et al. Environmental enrichment in adulthood promotes amblyopia recovery through a reduction of intracortical inhibition. Nature Neuroscience 2007; 10:679-81.

[61] Kolb B. Synaptic plasticity and the organization of behaviour after early and late brain injury. Canadian Journal of Experimental Psychology 1999;53:62-76.

[62] Anderson V, Spencer-Smith M, Wood A. Do children really recover better? Neurobehavioural plasticity after early brain insult. Brain 2011;134:2197-221.

[63] Ogunshola OO, Stewart WB, Mihalcik V, Solli T, Madri JA, Ment LR. Neuronal VEGF expression correlates with angiogenesis in postnatal developing rat brain. Brain Research Developmental Brain Research 2000;119:139-53.

[64] Song H, Stevens CF, Gage FH. Astroglia induce neurogenesis from adult neural stem cells. Nature 2002;417:39-44. 